\title{
Hahn-Steinthal Fracture: About 3 Cases
}

El Alami Réda*, Kadiri Mohammed, Driss Jeddi, Boufettal Mounef, Bassir Reda Allah, Kharmaz Mohammed, Lamrani Mly Omar, El Bardouni Ahmed, Mahfoud Mustapha, Berrada Mohamed Saleh

Department of Traumatology and Orthopedics, Ibn Sina Hospital, Rabat, Morocco

*Corresponding author: El Alami Réda

Abstract

Capitellum fractures are rare. Their initial management must be early and effective because of the risks generated on the elbow: rigidity, instability, arthritis. Many treatments have been proposed. The diagnosis is guided by clinical examination and confirmed on front, side and internal $3 / 4$ radiographs. The lesions are generally classified according to Bryan and Morrey. We report three cases of capitellum fractures. The patients underwent emergency surgery using Kocher posterolateral approach, open reduction and stabilization by Herbert screws. The average follow up period was one year. Total functional recovery was favorable. The functional evaluation was rated excellent according to the MEPI score (Mayo Elbow Performance Index) for the three patients. There was no dismantling of equipment. The average bone consolidation was 3 months.

Keywords: Capitellum, Hahn-Steinthal fracture, Herbert screwing, internal osteosynthesis.

Copyright @ 2020: This is an open-access article distributed under the terms of the Creative Commons Attribution license which permits unrestricted use, distribution, and reproduction in any medium for non-commercial use (NonCommercial, or CC-BY-NC) provided the original author and source are credited.

\section{INTRODUCTION}

Capitellum fracture is rare. It represents $1 \%$ of all elbow fractures and $6 \%$ of humerus fractures [1]. The initial management of these lesions must be early and effective because of the risks generated on the elbow in the short term: rigidity, instability; and those in the long term: post-traumatic arthritis [2]. Many treatments have been proposed [2]: radical treatment such as early excision of the capitellum and conservative treatment including osteosynthesis with different implants. Our cases describe the procedure using Herbert's screw in order to help improve the management of these lesions.

\section{PATIENTS AND OBSERVATIONS Patient 1}

A 34-year-old athletic man reported a history of closed trauma to the right elbow following a fall with reception on the palm of the hand two weeks earlier. He presented with pain and swelling of the right elbow, and limitation of flexion $\left(90^{\circ}\right)$. There was tenderness of the lateral aspect to the elbow.
The anatomical landmarks of the elbow were preserved, extension and prono-supination were free and there were no vascular or nervous disorders. Face and lateral radiographs (Fig-1) showed a vicious callus of the ascended capitellum in an anterohumeral position with no other associated lesions. Surgical reduction was performed by an external bicipital anterior way (Fig-2), with callus osteotomy, anatomical repositioning of the capitellum after preparation of the fixation site and excision of the fibrosis. The osteosynthesis was carried out by anteroposterior screwing in compression by a single capitulo-humeral screw. The reduction was stable with per operative gain on the elbow flexion by disappearance of the anterior stopper generated by the capitullum in vicious cal. Analgesic immobilization of the elbow by a brachio antebrachial splint was indicated. A week later, the pain was gone and the patient recovered good mobility of the elbow with flexion at $130^{\circ}$, extension at $0^{\circ}$, pronation at $90^{\circ}$, and supination at $90^{\circ}$. 


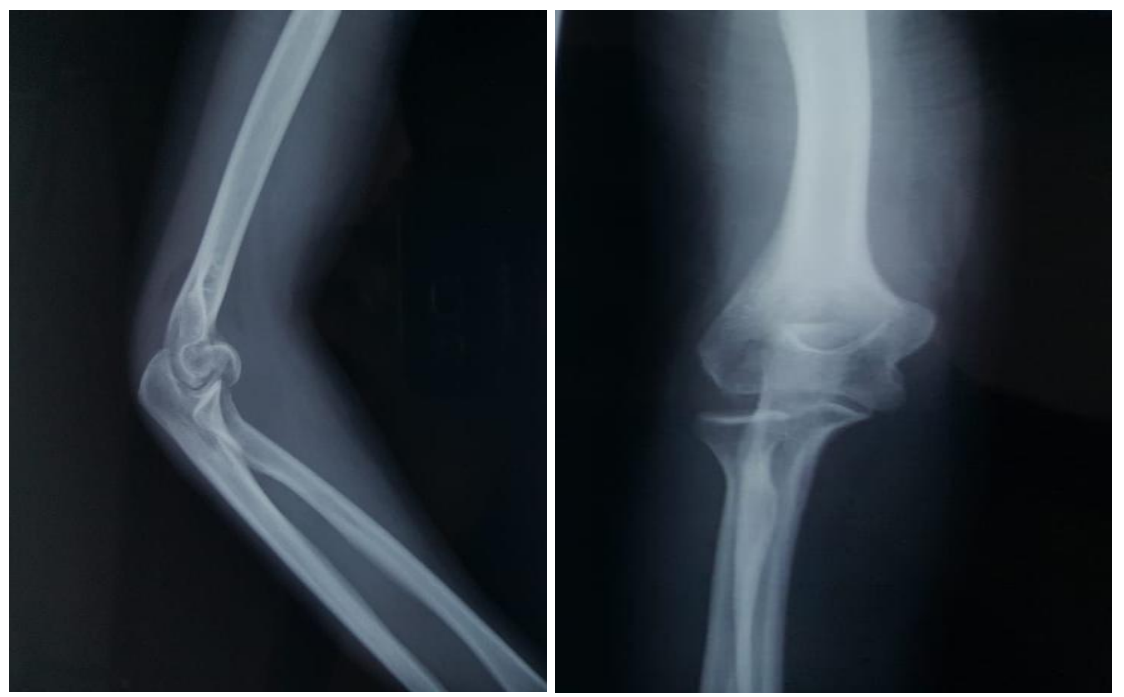

Fig-1: Lateral and front X-rays showing Capitellum fracture

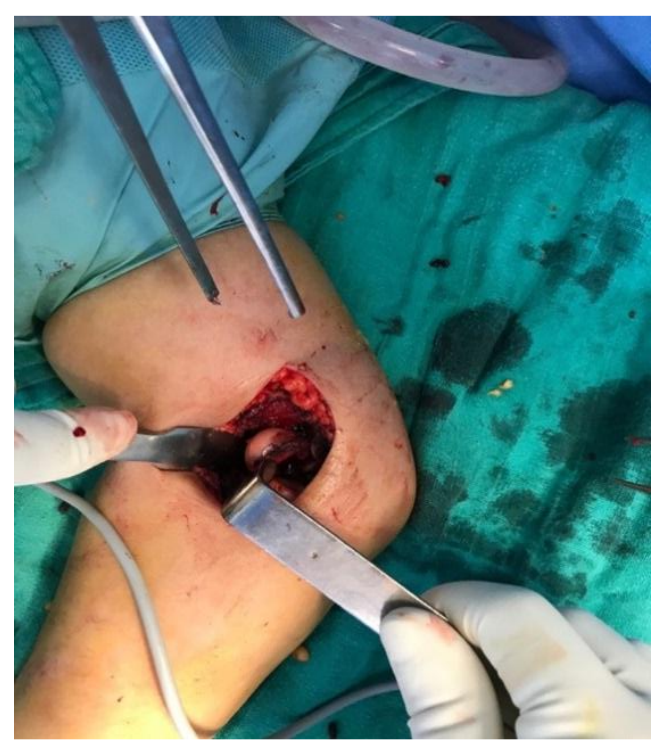

Fig-2: Peroperative picture showing the surgical approach

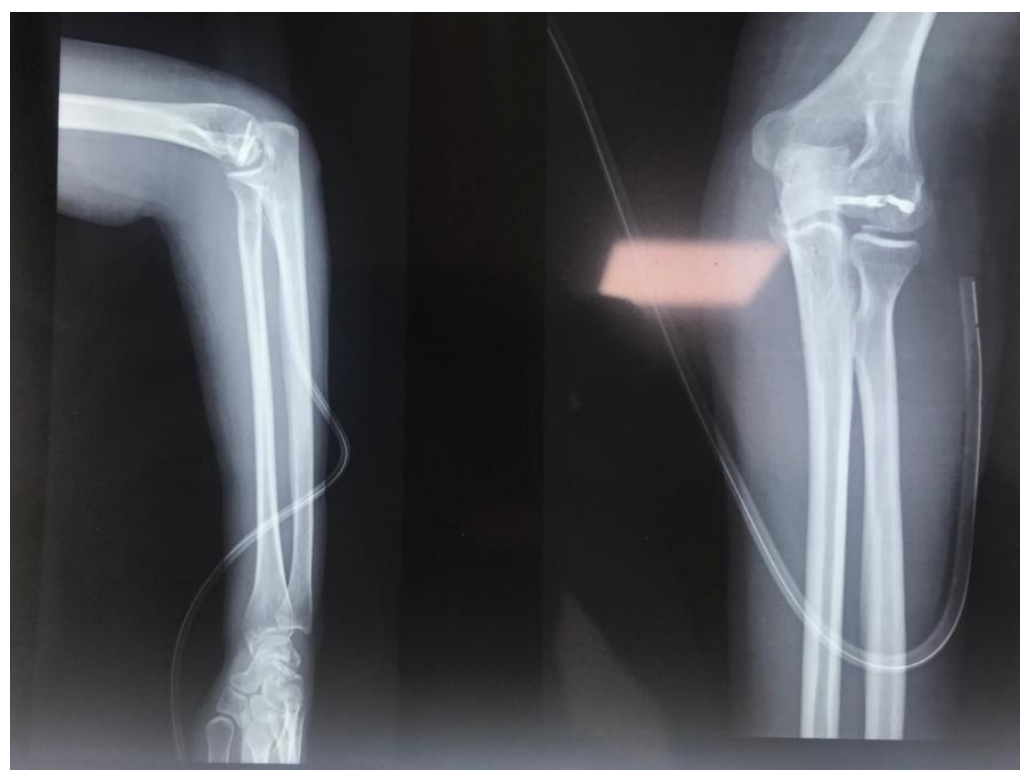

Fig-3: Postoperative front and lateral X-rays 


\section{Patient 2}

A 23-year-old right-handed woman sustained the injury in a fall from height with reception on the left elbow in flexion. She was admitted to the emergency room with pain and swelling of the left elbow. The radiographs showed a capitellum fracture extended to the lateral cheek of the humeral trochlea. The patient underwent open reduction through an extended lateral Kocher's approach, and the fracture was fixed with two extra-articular anteroposterior Herbert screws buried in the subchondral bone.

After checking the mobility of the elbow, additional immobilization of the elbow by posterior brachio-antebrachial splint was indicated for three weeks.

Reduced mobility was noted when the splint was removed. Rehabilitation was started a week later.

He regained a full range of movements at 3 months, and on follow up there were no radiological signs of arthritis or avascular necrosis (AVN). There was no dismantling of equipment. The MEPI score was 96.

\section{Patient 3}

A 21-year-old right-handed housewife, was admitted following a fall on the left elbow with pain and functional impotence.

Initial examination revealed a swollen, hot, painful elbow with exquisite anterolateral pain. The anatomical landmarks were preserved.

The radiographs (Fig-4) objectified a displaced fracture of the capitellum and the lateral cheek of the trochlea.

The patient underwent open reduction and internal osteosynthesis according to the same principles.

Functional recovery was obtained after 3 months with a MEPI score of 98 . Bone consolidation was complete at 3 months. There was no dismantling of equipment.
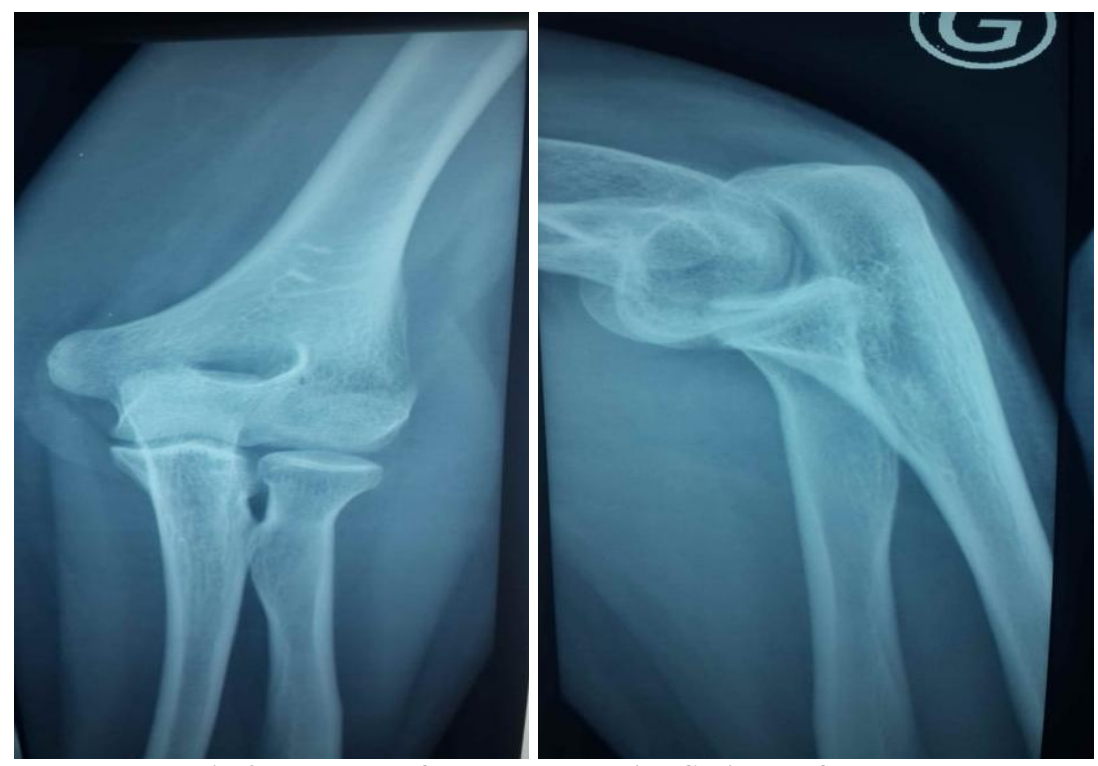

Fig-4: Lateral and front X-rays showing Capitellum fracture

\section{DISCUSSION}

Capitellum fractures are rare injuries that occur in adolescents over the age of 12 years. Though, reportedly, more common in females, with a male to female ratio of 1:4.

They are frontal articular parcel fractures of the humeral palette. The fracture line sits in the frontal plane and detaches an anterior fragment of the articular cartilaginous epiphysis of lateromedial extent and of variable thickness.

The different types of these fractures continue to be included under the vague term of frontal fractures of the capitellum whereas the classifications and the eponyms frequently used to designate them are not unequivocal and can induce confusion [3, 4]. The favoring factors encountered are cubitus-valgus and cubitus-recurvatum [5].

Grantham et al., [6] had identified three types of frontal fractures of the capitellum according to the thickness and the comminution of the osteochondral articular fragment released by the line:

- Type I is a separation of a thin cartilaginous fragment deprived of its bony base;

- Type II is a cartilaginous fragment with a thick underlying bone base;

- Type III is a comminuted fracture of the capitellum.

Bryan and Morrey Classification [6] distinguishes three types: 
- Type I, known as the Hahn-Steinthal fracture: detaches a large bone fragment comprising the entire condyle and possibly part of the external trochlear cheek;

- Type II, known as the Kocher-Lorenz or Mouchet fracture; it is a small fragment, sometimes osteochondral, consisting of a more or less important part of the condyle;

- Type III, known as Kocher's diacondylar fracture, takes the entire articular mass up to the internal bank of the trochlea.

Patients usually present with pain and lateral swelling of the elbow after a medium energy trauma. The causes described in the literature, and found in our patients are falls on the bent elbow. This results from the transmission of an axial force through the radius which shears the capitulum in the coronal plane [6].

The diagnosis is based on lateral radiographs showing a fragment in the shape of "crescent of the Moon" detached from the humeral condyle.

A CT with possible reconstruction can be carried out to define the size of the fragment and the operative planning but it was unnecessary in our cases.

Closed reduction and plastered immobilization were reported [7]. Hahn was the first to report the unsatisfactory outcome of orthopedic treatment for capitellum fractures.

During the autopsy, he found that the capitellum had been moved and united with the anterior aspect of the humerus causing the restriction of the elbow flexion [8].

Our treatment consisted of an open reduction and stabilization with 2 buried Herbert screws, thus allowing better compression. A mobility testing was done intraoperatively, assessing congruence.

A wide variety of internal stabilization techniques have been described, such as Kirschner wires, biodegradable pins, staples, bone clamps, and compression screws [9]. The pins do not provide strong compression and stabilization of the fracture site.

Most of these methods require a long period of post-operative immobilization which interferes with early functional recovery.

Herbert's screws have the advantage of being buried so that they do not irritate the soft tissue and removal of the implants is unneeded. Therefore, the rehabilitation program can start earlier and functional recovery is better.

The MEPI score for our patients was globally excellent, reflecting good functional recovery.
Open focus reduction and internal fixation (ORIF of Anglo-Saxons) is an appropriate method to maintain common congruence while allowing early mobilization. Kirschner wire and AO compression screws are the most widely used materials in the literature, even if the current trend leans towards the use of cannulated and headless screws [8]. Kirschner wires do not provide compression at the fracture site and require subsequent removal. The $\mathrm{AO}$ screws meet the main requirements for this kind of fracture, namely compression even if they can irritate the cartilage of the radial head due to exposed screws.

The most widely used approach in the literature is the lateral approach which allows reduction, fixation and osteosynthesis of the bone fragment [10]. For our patients, the choice of the anterior approach, with all the risks described, was mainly motivated by the unusual variety of displacement of the bone fragment antehumerally, making any other approach initially laborious and uncertain.

Headless screws can have problems if the patients develop AVN or chondrolysis, because erosion of the radial head is a possibility due to exposed implants. This problem is avoided by the $4 \mathrm{~mm}$ partially threaded screws. Reports of avascular necrosis of the capitellum are very rare [11].

Our patients showed no sign of osteonecrosis despite the fact that there was soft tissue fixation and without any significant bone support to revascularize the fragment. The literature suggests that if osteonecrosis does not occur within a year, it is unlikely to occur later [12]. McKee et al. reported a patient suffering from post-traumatic osteoarthritis in his series of six patients [13]. In a large series, Lansinger et al., found no cases of osteoarthritis of the elbow [14]. We did not find osteoarthritis in our patients at the last follow-up and we believe that if the reduction is anatomical with minimal damage to the articular cartilage, osteoarthritis can be avoided.

\section{CONCLUSION}

Open reduction and internal fixation are the treatment of choice for the Hahn Steinthal fractures. Stabilization using a Herbert screw provides good results because it allows strong inter-fragmentary compression, early mobilization, and thus functional recovery of the elbow. Removal of osteosynthesis material is rarely necessary.

\section{REFERENCES}

1. Mehdian H, McKee MD. Fractures of capitellum and trochlea. Orthop Clin North Am. 2000;31(1):127.

2. Bryan RS, Morrey BF. Fractures of the distal humerus. In: Morrey BF, editor. The Elbow and its 
Disorders. Philadelphia: Saunders W B; 1985;302-39.

3. Lecestre P, Aubaniac JM, Claisse P, Copin G., Dupont JY, Duriau F. Les fractures de l'extrémité inférieure de l'humérus chez l'adulte Rev Chir Orthop 1980; 66 (Suppl. II) : 21-50 Hachimi K., Hattoma N., Sennoune B., Rafai M., Largab A., Trafeh M. Traitement chirurgical des fractures du capitellum chez l'adulte. À propos de huit cas Chir Main, 2004;23:79-84

4. Fenoll B. Fractures du capitellum Traumatologie du coude de l'enfant Paris: Elsevier, 2000;92-99

5. Kocher T. 3rd edition. London: Adam and Charles Black; 1911. Textbook of operative surgery.

6. Morrey BF, An KN. Functional evaluation of the elbow. In: Morrey BF, Sanchez-Sotelo J, editors. The elbow and its disorders. fourth edition. Philadelphia: Saunders W B; 2009;80-91.

7. Letts M, Rumball K. Fractures of the capitellum in adolescents. Journal Pediatr Orthop. 1997;17(3):315-20.

8. Hahn NF. Fall von einer besonderen Varietat der Frakturen des Ellenbogens. Zeitschrift fur Wundarzte und Geburtshelfer. 1853;6:185-9.
9. Pogliacomi F, Concari G, Vaienti E. HahnSteinthal fracture: report of two cases. Acta Biomed. 2005;76(3):178-84.

10. Nawghare SP, Baidyaray R, Neyt JGV. HahnSteinthal fracture: a case report. Cases Journal. 2008;1:239.

11. Schindler OS. Bilateral capitellum humeri fracture: a case report and review of the literature. J Orthop Surg. 2003;11(2):207-12.

12. Mason ML. Some observations on fractures of the head of the radius with a review of one hundred cases. Br J Surg. 1954;41(172):123-32.

13. Dubberley JH, Faber KJ, Macdermid JC, Patterson SD, King GJW. Outcome after open reduction and internal fixation of capitellar and trochlear fractures. Journal Bone Joint surg. 2006;88A:4654.

14. McKee MD, Jupiter JB, Bamberger HB. Coronal shear fractures of the distal end of the humerus. J Bone Joint Surg Am. 1996 Jan;78(1):49-54.

15. Lansinger O, Mare K. Fracture of the capitellum humeri. Acta Orthop Scand. 1981;52:39-44. 